\title{
Tratamento de fístula bucosinusal após exodontia com corpo adiposo da bochecha e retalho vestibular em paciente diabético: relato de caso
}

Treatment of oroantral fistula after extraction with buccal fat pad and buccal flap in a diabetic patient: case report Tratamiento de fístula bucosinusal después de extracción con cuerpo adiposo de mejilla y colgajo en paciente diabético: reporte de caso Rodrigo CAPALBO-SILVA ${ }^{1}$

Hiskell Francine Fernandes e OLIVEIRA ${ }^{2}$ Henrique HADAD ${ }^{1}$

Bruno Coelho MENDES ${ }^{1}$

Breno dos Reis FERNANDES ${ }^{1}$

Lara Cristina Cunha CERVANTES ${ }^{1}$ Francisley Ávila SOUZA ${ }^{1}$

${ }^{1}$ Department of Diagnostic and Surgery - São Paulo State University, Araçatuba School of Dentistry, Araçatuba, Brazil ${ }^{2}$ Department of Dental Materials and Prosthodontics - São Paulo State University, Araçatuba School of Dentistry, Araçatuba, Brazil

\section{Resumo}

A comunicação bucosinusal trata-se da comunicação não natural da cavidade bucal com o seio maxilar, estando muitas vezes relacionada a extração dos dentes superiores posteriores. A literatura apresenta diversas opções de tratamento para esses casos, entre eles o fechamento com o retalho pediculado com o corpo adiposo bucal. O objetivo deste trabalho foi relatar um caso de fístula bucosinusal em paciente diabético, discutindo alternativas cirúrgicas correlacionadas com problema sistêmico do paciente e características locais do defeito. Paciente do sexo masculino, 55 anos de idade, com histórico de dez dias de exodontia do elemento 27, com queixa de passagem de ar ao meio bucal através do sítio cirúrgico. Com base nos exames, o diagnóstico definitivo foi de comunicação bucosinusal, sendo estipulado o tratamento cirúrgico para o fechamento da comunicação através de duas camadas com o corpo adiposo da bochecha seguido do retalho vestibular. No acompanhamento de 8 meses e meio o paciente não apresenta queixas e pode-se observar o fechamento completo da comunicação bucosinusal. O retalho pediculado do corpo adiposo bucal seguido do retalho vestibular mostrou-se efetivo no tratamento da fístula bucosinusal em paciente diabético controlado.

Descritores: Fístula Bucoantral; Cirurgia Bucal; Diabetes Mellitus.

\section{Abstract}

Oroantral communication is the unnatural communication of the oral cavity with the maxillary sinus and is often related to the extraction of the upper posterior teeth. The literature presents several treatment options for these cases, including closure with the pedicled flap with the buccal fat pad. The aim of this paper was to report a case of an oroantral fistula in a diabetic patient, discussing surgical alternatives correlated with the patient's systemic disease and local characteristics of the defect. A 55-year-old male patient has a history of the 27 tooth extraction 10 days ago. He has complaining of air passing through the surgical site to the oral cavity. Based on the exams, the definitive diagnosis was oroantral communication and surgical treatment was stipulated to close the communication through two layers with the buccal fat pad followed by the buccal flap. At 8 and half months of follow-up the patient has no complaints and the complete closure of the oroantral communication can be observed. The pediculated buccal fat pad flap followed by the buccal flap proved to be effective in the treatment of oroantral fistula in a controlled diabetic patient.

Descriptors: Oroantral Fistula; Surgery, Oral; Diabetes Mellitus.

\section{Resumen}

La comunicación oroantral es la comunicación no natural de la cavidad oral con el seno maxilar, y a menudo está relacionada con la extracción de los dientes superiores posteriores. La literatura presenta varias opciones de tratamiento para estos casos, incluido el cierre con el colgajo pediculado cuerpo adiposo de la boca. El objetivo de este trabajo es informar un caso de fístula oroantral na en un paciente diabético, discutiendo alternativas quirúrgicas relacionadas con la enfermedad sistémica del paciente y las características locales del defecto. Paciente masculino de 55 años con antecedentes de extracción del elemento 27 durante 10 días, quejándose de que el aire pasa a través del sitio quirúrgico hacia la cavidad oral. Según los exámenes, el diagnóstico definitivo fue comunicación bucal y sinusal, y se estipuló el tratamiento quirúrgico para cerrar la comunicación a través de dos capas con el cuerpo adiposo de la boca por el colgajo bucal. A los 8 meses y medio de seguimiento, el paciente no tiene quejas y se puede observar el cierre completo de la comunicación bucosinusal. El colgajo de almohadilla de grasa bucal pediculada seguido del colgajo bucal demostró ser efectivo en el tratamiento de la fístula oroantral en un paciente diabético controlado.

Descriptores: Fístula Oroantral; Cirugía Bucal; Diabetes Mellitus.

INTRODUÇÃO

$\mathrm{O}$ seio maxilar se destaca entre os seios paranasais por apresentar o maior volume, podendo estar em íntimo contato com os ápices radiculares dos dentes posteriores superiores ${ }^{1}$. Essa característica anatômica permite que em algumas situações clínicas como exodontia, periodontopatias apicais, remoções de lesões e traumas haja uma comunicação não natural da cavidade bucal com o seio maxilar, denominada comunicação bucosinusal ${ }^{2}$. Quando não tratada dentro do período de 48 a 72 horas, a comunicação bucosinusal se torna fístula devido a formação de um canal epitelial neste trajeto ${ }^{3}$.

Clinicamente a fístula bucosinusal pode se manifestar em sinais e sintomas como passagem de ar ou fluidos do seio maxilar para a boca, dor, alteração fonética e gosto ruim na boca relatado pelo paciente.
A comunicação bucosinusal pode ser confirmada com a manobra de Valsalva, que consiste na pressão positiva de ar gerada pelo paciente contra as narinas fechadas, havendo o escape de ar pela fístula muitas vezes gerando um ruído sibilante ${ }^{4}$. Radiograficamente, onde o exame complementar ideal é a tomografia computadorizada, a comunicação bucosinusal caracterização pela descontinuidade do assoalho do seio maxilar, podendo haver o espessamento da mucosa do seio em casos crônicos bem como sinusopatias ${ }^{1}$. Deve-se descartar a presença de processos inflamatórios ou infecciosos no seio maxilar, devendo ser tratados previamente ao procedimento cirúrgico quando presentes ${ }^{5}$.

A literatura apresenta várias resoluções cirúrgicas para o tratamento das comunicações 
bucosinusais baseadas principalmente no tamanho do defeito, tempo de diagnóstico, epitelização do trajeto, presença de infecção no seio maxilar, e tensão necessária para o fechamento por primeira intensão da ferida cirúrgica, não havendo um consenso na efetividade de cada técnica ${ }^{6-8}$. Esses fatores mostram a importância de um correto exame clínico e o suporte dos exames de imagem que confirmam o padrão da comunicação. Três tipos de retalho são os mais preconizados para o fechamento das comunicações oroantrais: o retalho vestibular, retalho palatino e o fechamento através do corpo adiposo da bochecha pediculado.

O retalho vestibular é o método mais utilizado para o tratamento das comunicações oroantrais devido a sua técnica de fácil execução e previsibilidade quando bem executado, entretanto apresenta limitações, sendo indicado para comunicações com menor diâmetro ${ }^{9-11}$. Um aspecto que deve ser analisado para esta técnica é se haverá tecido suficiente para o fechamento por primeira intenção sem gerar muita tensão sobre o retalho a fim de evitar isquemia e necrose tecidual. Incisões horizontais no periósteo permitem uma melhor passividade do retalho, entretanto o retalho vestibular tem como característica a perda de fundo de vestíbulo devido à tensão causada na região, que muitas vezes deve ser corrigida através de sulcoplastias posteriormente para reabilitações protéticas das áreas afetadas?.

O retalho palatino é uma alternativa para o fechamento de fístulas bucosinusais bem estabelecida $^{12}$. Apresenta variações de acordo com sua espessura e desenho para o seu deslocamento. É indicado para defeitos maiores e tardios, diferentemente dos defeitos indicados para o retalho vestibular. Como vantagens apresenta a boa irrigação quando a artéria palatina é envolvida ao retalho, a manutenção do fundo de vestíbulo, e qualidade do tecido por se tratar de um tecido altamente queratinizado $^{3}$. Como desvantagem pode apresentar complicações como hemorragia trans-operatória por erro de técnica cirúrgica, necrose do retalho, dor pósoperatória devido a área cruenta que fica no palato, e irregularidade na superfície do palato devido a cicatrização por segunda intensão no leito doador ${ }^{9}, 13$.

$\mathrm{O}$ enxerto pediculado do corpo adiposo bucal é outra opção de tratamento para esses casos. Está indicado para comunicações com defeitos com diâmetro maior que $5 \mathrm{~mm}^{14}$, não alterando função ou estética para o paciente. O corpo adiposo da bochecha possui fácil acesso cirúrgico e considerável mobilidade, o que permite seu tracionamento e posicionamento sobre a fístula, que associado a sua boa vascularização se torna uma ótima alternativa para o fechamento de comunicações bucosinusais ${ }^{15}$. Sua técnica consiste no descolamento da mucosa vestibular e o pinçamento do corpo adiposo da bochecha por baixo deste retalho seguido de seu tracionamento para a área da comunicação e sutra na mucosa palatina. O processo de epitelização é evidenciado na cavidade oral no sétimo dia pósoperatório e é completa na $3^{\mathrm{a}}$ ou $4^{\mathrm{a}}$ semana por um processo de metaplasia ${ }^{16}$. Estudos mostram altas taxas de sucesso desta técnica, bem como sua variação com a associação a outras camadas durante o fechamento como o retalho vestibular ${ }^{3,4,7,8,16}$. Diante do exposto, o objetivo deste relato de caso é apresentar o fechamento de uma fístula bucosinusal através do retalho pediculado do corpo adiposo bucal abordando diagnóstico e técnica cirúrgica.

\section{CASO CLÍNICO}

Paciente do sexo masculino, 55 anos de idade, foi encaminhado à Faculdade de Odontologia de Araçatuba aos cuidados da equipe de Cirurgia e Traumatologia Bucomaxilofacial, com histórico, dez dias antes, de exodontia do elemento 27 , com queixa de passagem de ar ao meio bucal através do sítio cirúrgico. Paciente relatava ter diabetes do tipo II, fazer uso regular de hipoglicemiante oral, e estar sob prescrição amoxicilina + clavulanato de potássio desde o dia da exodontia. Ao exame físico pode-se notar o alvéolo correspondente ao elemento 27 recém-extraído em processo de reparo com grande comunicação com o seio maxilar (Figura 1).

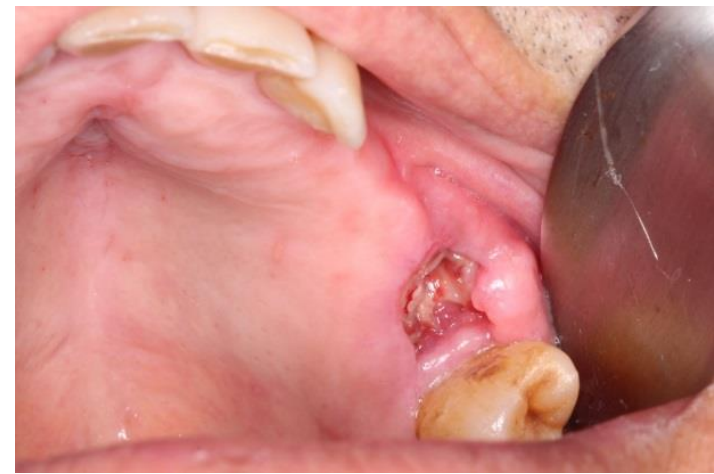

Figura 1: Alvéolo em fase de reparo inicial de 10 dias após extração de elemento 27 com comunicação bucosinusal.

Durante a realização da manobra de Valsalva foi observada passagem de ar pelo alvéolo com presença de secreção advinda do seio maxilar. Ao exame de imagem (radiografia panorâmica e tomografia computadorizada), observou-se uma descontinuidade do assoalho do seio maxilar na região do elemento dentário extraído com um defeito de cerca de $7 \mathrm{~mm}$ de diâmetro (Figura 2). Com base nos exames, o diagnóstico definitivo foi de comunicação bucosinusal, sendo estipulado o tratamento cirúrgico ambulatorial para o fechamento da comunicação através de duas camadas com o corpo adiposo da bochecha seguido do retalho vestibular. Paciente recebeu administração de acetilcisteína 3 dias previamente ao procedimento cirúrgico para diminuição da secreção e antibioticoterapia profilática. 


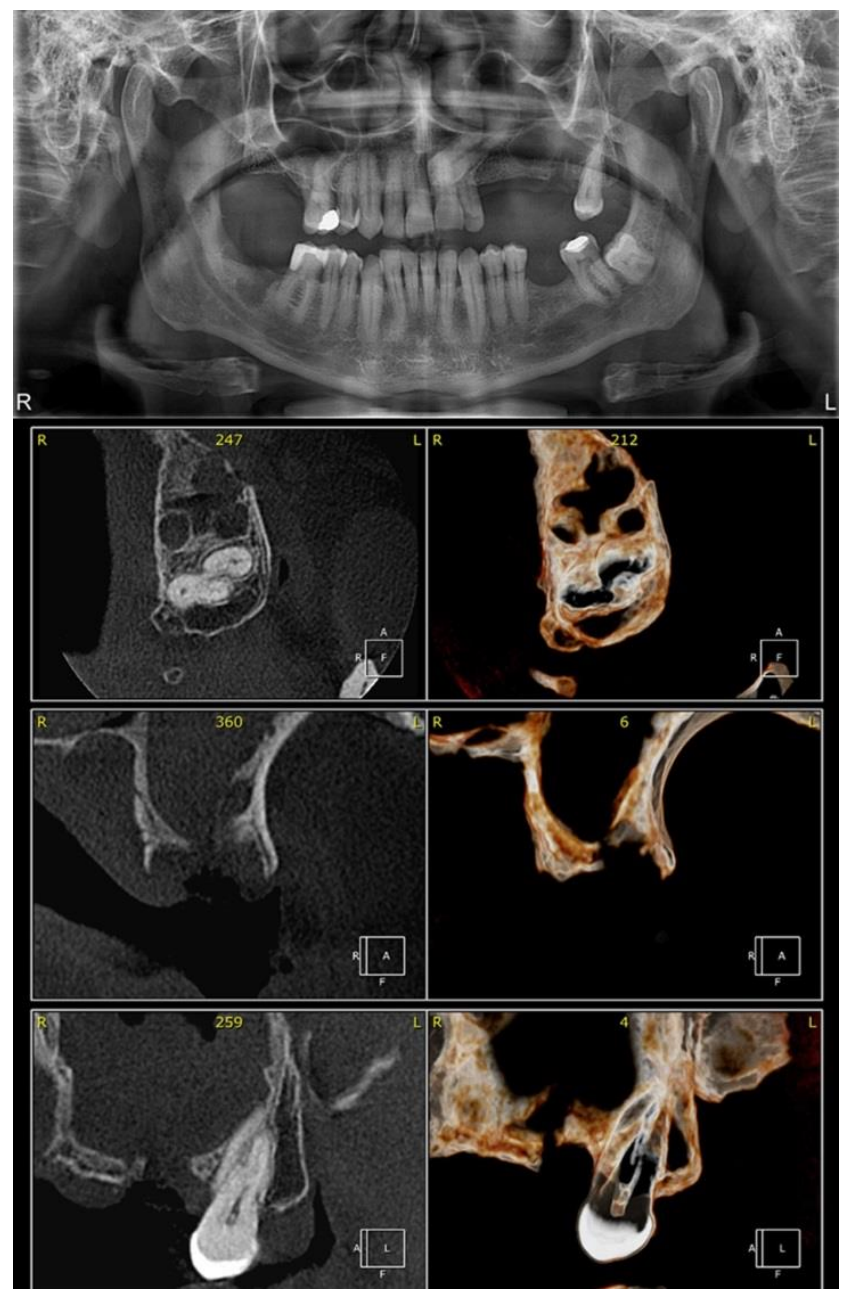

Figura 2: Radiografia panorâmica e tomografia computadorizada evidenciando descontinuidade de assoalho de seio maxilar em região de elemento 27.

Foi realizada antissepsia intraoral com clorexidina $0,12 \%$ e extraoral com clorexidina $2 \%$ e aposicionamento do campo fenestrado. Foi realizada anestesia dos nervos alveolares superiores posteriores, médios e palatino maior do lado esquerdo. Procedeu-se com incisão linear sobre o rebordo associada a uma incisão oblíqua distante do local da comunicação para melhor liberação do retalho. Durante a incisão realizou-se a remoção do cordão epitelial formado entre o meio bucal e o seio maxilar (Figura 3), seguido do descolamento mucoperiosteal da região vestibular para o acesso ao corpo adiposo da bochecha. Com o deslocamento do retalho, através de uma pinça halstead mosquito curva os tecidos foram divulcionados para que o corpo adiposo da bochecha fosse encontrado. Após isso, com auxílio de uma pinça Dietrich a bola de Bichat foi tracionada de modo que preenchesse todo o defeito (Figura 4) e foi suturada à mucosa palatina com fio nylon 4-0 (Figura 5). Após a estabilização do corpo adiposo procedeu-se com a liberação do retalho vestibular através de incisões horizontais no periósteo na base do retalho permitindo que o fechamento da ferida cirúrgica por primeira intenção sem tracionamento excessivo dos tecidos viabilizando a irrigação adequada para um melhor processo cicatricial (Figura 6). A sutura do retalho vestibular foi realizada com fio de nylon 5-0, resultando no fechamento primário do sítio cirúrgico. $\mathrm{O}$ paciente foi orientado quanto aos cuidados pós-operatórios e recebeu prescrição de antibiótico, anti-inflamatório não esteroidal, analgésico e permaneceu com o uso de acetilcisteína.

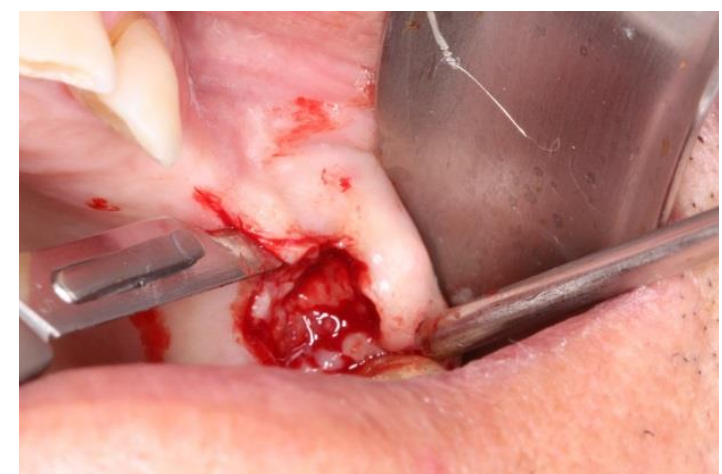

Figura 3: Remoção do cordão epitelial formado da fístula e incisão para liberação do retalho vestibular.

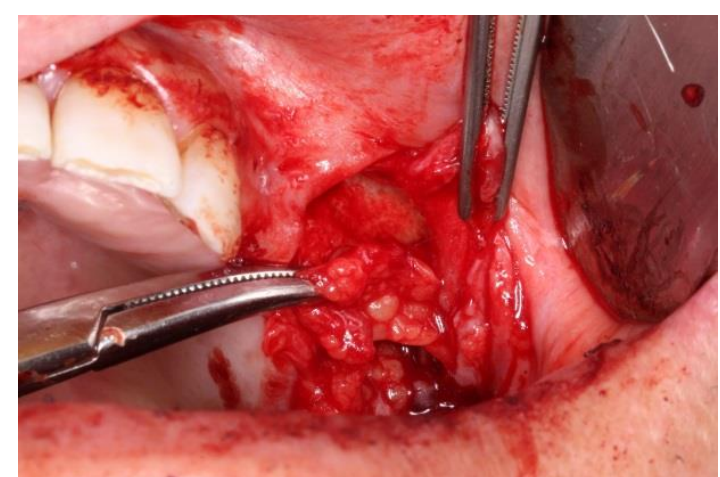

Figura 4: Corpo adiposo bucal pediculado sendo tracionado na área do defeito para sua obliteração.

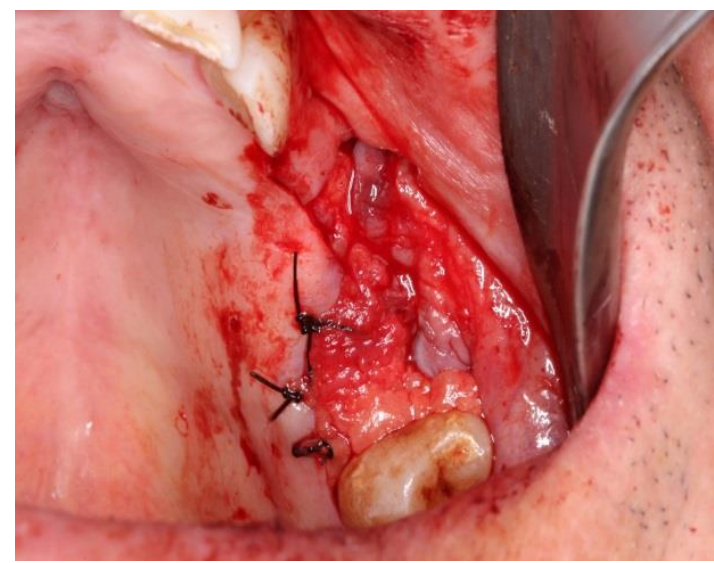

Figura 5: Corpo adiposo bucal suturado em mucosa palatina.

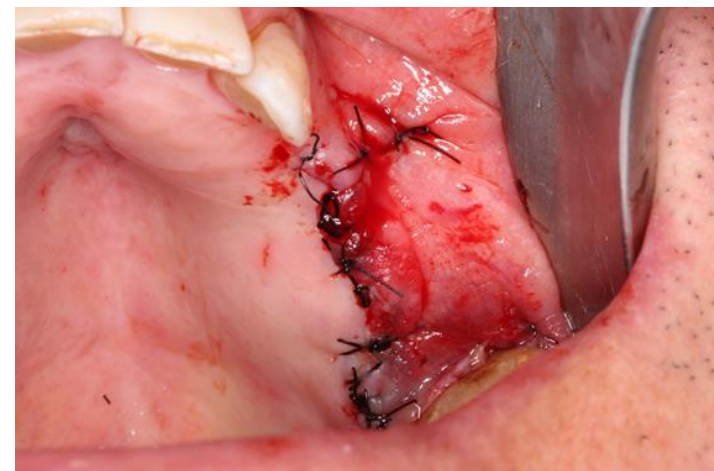

Figura 6: Retalho vestibular recobrindo corpo adiposo bucal como segunda camada. 
O acompanhamento pós-operatório semanal do paciente foi realizado no primeiro mês rigorosamente, onde o mesmo foi orientado continuamente com os cuidados necessários. No acompanhamento de 8 meses e meio o paciente não apresenta queixas e pode-se observar o fechamento completo da comunicação bucosinusal (Figura 7) e ausência de sinais ou sintomas de sinusite.

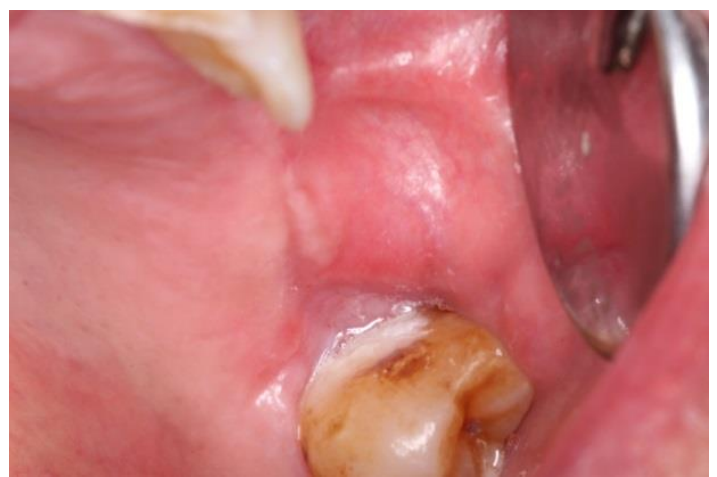

Figura 7: Acompanhamento de 8 meses e meio com fechamento completo da comunicação bucosinusal.

\section{DISCUSSÃO}

A associação entre anamnese criteriosa e exame físico, suportados por exames complementares permitem o diagnóstico preciso, direcionando a escolha correta no tipo de tratamento das comunicações bucosinusais, consequentemente promovendo um aumento em suas taxas de sucesso ${ }^{4}$.

A condição sistêmica do paciente pode influenciar na eleição do tratamento cirúrgico. Erdoğan et al. ${ }^{13}$ relataram a necrose tecidual em área doadora durante o fechamento de comunicação bucosinusal através do retalho palatino em paciente diabético. A necrose na região palatina é uma complicação comum a esta técnica cirúrgica podendo ser potencializada na presença de comorbidades sistêmicas que favorecem o atraso cicatricial e infecções como a diabetes. Esse fator associado ao tamanho do defeito do presente caso foi decisivo na escolha do fechamento através do retalho pediculado do corpo adiposo bucal.

Estudos indicam que a o retalho pediculado com o corpo adiposo da bochecha está indicado para defeitos de maiores diâmetros apresentando alta taxa de sucesso em seu tratamento ${ }^{3,4,9}$. Poeschi et al. ${ }^{17}$ relatam uma taxa de sucesso de $98 \%$ dos casos no fechamento de comunicação bucosinusal através dessa técnica, sendo o maior defeito com o tamanho de $56 \times 42 \mathrm{~mm}$ criado após ressecção de tumor. Mostrando que, para defeitos menores como no caso das comunicações por exodontia, é um tratamento com boa previsibilidade.

O uso da técnica do retalho pediculado do corpo adiposo associado ao retalho vestibular é utilizado na tentativa de promover um fechamento mais seguro e previsível por apresentar uma camada a mais. Batra et al. ${ }^{18}$ por meio de um estudo clínico compararam as duas técnicas de forma isolada e sua associação, tendo complicações apenas na técnica do retalho vestibular isolada, não havendo diferença entre a exposição do corpo adiposo da bochecha e o seu recobrimento com o retalho bucal com o fechamento de todos os casos. Entretanto alguns estudos defendem o fechamento através de mais de uma camada ${ }^{19,20}$.

CONCLUSÃO

Conclui-se que, a anamnese criteriosa acompanhada do correto diagnóstico da comunicação bucosinusal baseado em tamanho do defeito, tempo, presença de infecção no seio maxilar, e tensão necessária para o seu fechamento, permitem a seleção do tratamento adequado para o caso. $\mathrm{O}$ retalho pediculado do corpo adiposo bucal mostrou-se efetivo no tratamento da fístula bucosinusal maior que $5 \mathrm{~mm}$ em paciente diabético controlado.

\section{REFERÊNCIAS}

1. Lozano-Carrascal N, Salomó-Coll O, Gehrke SA, Calvo-Guirado JL, Hernández-Alfaro F, GargalloAlbiol J. Radiological evaluation of maxillary sinus anatomy: A cross-sectional study of 300 patients. Ann Anat. 2017;214:1-8.

2. Jang JK, Kwak SW, Ha JH, Kim HC. Anatomical relationship of maxillary posterior teeth with the sinus floor and buccal cortex. J Oral Rehabil. 2017;44(8):617-25.

3. Khandelwal P, Hajira N. Management of Oroantral Communication and Fistula: Various Surgical Options. World J Plast Surg. 2017;6(1):3-8.

4. Parvini P, Obreja K, Begic A, et al. Decisionmaking in closure of oroantral communication and fistula. Int J Implant Dent. 2019;5(1):13.

5. Lin PT, Bukachevsky R, Blake M. Management of odontogenic sinusitis with persistent oro-antral fistula. Ear Nose Throat J. 1991;70(8):488-90.

6. Al-Juboori MJ, Al-Attas MA, Magno Filho LC. Treatment of chronic oroantral fistula with platelet-rich fibrin clot and collagen membrane: a case report. Clin Cosmet Investig Dent. 2018; 10:245-49.

7. Kiran Kumar Krishanappa S, Eachempati P, Kumbargere Nagraj S, Shetty NY, Moe S, Aggarwal $\mathrm{H}$ et al. Interventions for treating oroantral communications and fistulae due to dental procedures. Cochrane Database Syst Rev. 2018;8(8):CD011784.

8. Darr A, Jolly K, Martin T, Monaghan A, Grime P, Isles $\mathrm{M}$ et al. Three-layered technique to repair an oroantral fistula using a posterior-pedicled inferior turbinate, buccal fat pad, and buccal mucosal advancement flap. Br J Oral Maxillofac Surg. 2018;56(7):638-39.

9. Parvini P, Obreja K, Sader R, Becker J, Schwarz F, Salti L. Surgical options in oroantral fistula management: a narrative review. Int J Implant Dent. 2018;4(1):40. 
10. Lin PT, Bukachevsky R, Blake M. Management of odontogenic sinusitis with persistent oro-antral fistula. Ear Nose Throat J. 1991;70(8):488-90.

11. Borgonovo AE, Berardinelli FV, Favale M, Maiorana C. Surgical options in oroantral fistula treatment. Open Dent J. 2012;6:94-8.

12. Ribeiro FS, de Toledo CT, Aleixo MR, Durigan MC, Silva WC, Bueno SK et al. Treatment of Oroantral Communication Using the Lateral Palatal Sliding Flap Technique. Case Rep Med. 2015;2015:730623.

13. Erdoğan $\mathrm{O}$, Esen E, Ustün Y. Bony palatal necrosis in a diabetic patient secondary to palatal rotational flap. J Diabetes Complications. 2005;19(6):364-67.

14. Tideman H, Bosanquet A, Scott J. Use of the buccal fat pad as a pedicled graft. J Oral Maxillofac Surg. 1986;44(6):435-40.

15. Yang S, Jee YJ, Ryu DM. Reconstruction of large oroantral defects using a pedicled buccal fat pad. Maxillofac Plast Reconstr Surg. 2018; 40(1):7.

16. Raldi FV, Sardinha SCS, Albergaria-Barbosa JR. Fechamento de comunicação bucossinusal usando enxerto pediculado com corpo adiposo bucal. BCI. 2000;7(25):60-3.

17. Poeschl PW, Baumann A, Russmueller G, Poeschl E, Klug C, Ewers R. Closure of oroantral communications with Bichat's buccal fat pad. J Oral Maxillofac Surg. 2009;67(7):1460-66.

18. Batra H, Jindal G, Kaur S. Evaluation of different treatment modalities for closure of oro-antral communications and formulation of a rational approach. J Maxillofac Oral Surg. 2010;9(1):13-8.

19. Weinstock RJ, Nikoyan L, Dym H. Composite three-layer closure of oral antral communication with 10 months follow-up-a case study. J Oral Maxillofac Surg. 2014;72(2):266.e1-266.e2667.

20. Candamourty R, Jain MK, Sankar K, Babu MR. Double-layered closure of oroantral fistula using buccal fat pad and buccal advancement flap. J Nat Sci Biol Med. 2012;3(2):203-5.

\section{CONFLITO DE INTERESSES}

Os autores declaram não haver conflitos de interesse.

\section{AUTOR PARA CORRESPONDÊNCIA}

\section{Rodrigo Capalbo da Silva}

Rua José Bonifácio, 1193, Vila Mendonça

16015-050 Araçatuba - São Paulo, Brasil

Telefone: +55 18 3636-2898

E-mail: capalbo.rodrigo@gmail.com

Submetido em 13/09/2019

Aceito em 07/08/2020 